\title{
Endovascular Repair of Blunt Traumatic Thoracic Aortic Injuries
}

\author{
Mehrdad Vahedian, ${ }^{1}$ Somayeh Rastinnia,, ${ }^{1, *}$ and Masumeh Taghizadeh ${ }^{1}$ \\ ${ }^{1}$ Department of General Surgery, Bahonar Hospital, Kerman University of Medical Sciences, Kerman, IR Iran \\ ${ }^{*}$ Corresponding author: Somayeh Rastinnia, Department of General Surgery, Bahonar Hospital, Kerman University of Medical Sciences, Kerman, IR Iran. Tel: +98-9177152306, \\ Fax: +98-3432221600, E-mail: arttemis17@yahoo.com
}

Received 2013 January 16; Revised 2015 June 15; Accepted 2015 July 15.

Keywords: Thoracic Aorta, Stent Graft, Thoracic Injury, Thorasic Endovascular Aortic Repair

\section{Dear Editor,}

During the last decade, endovascular (EV) repair has replaced open surgical repair (OSR) as the preferred method of treatment of blunt traumatic thoracic aortic injuries (BTAIs) at many trauma centers. The thoracic endovascular aortic repair (TEVAR) means percutaneous replacement of a stent within the descending thoracic or thoracoabdominal aorta to treat aortic aneurysm (1). The thoracic endovascular aortic repair has the advantage of being a less invasive technique compared to the open surgical method of repairing, owing to the unique ability to insert the stent through a small incision. Because the incision is small, the patients who are operated by the TEVAR technique have minimal amounts of blood loss. Through the TEVAR method, prolonged cross clamping of aorta is not necessary. As a result, the incidence of renal, visceral and spinal ischemia is fewer than that in the standard open surgical repair technique (2). Studies show that the TEVAR reduces early mortality and paraplegia compared with the open surgical management. The risk of dependency to the mechanical respiratory ventilation is lower in the thoracic endovascular aortic repairmen. Unlike the traditional aortic repair, standard recovery after the TEVAR is remarkably straightforward. Patients, who have undergone the TEVAR, typically spend one night in hospital to be monitored although it has been suggested that the TEVAR can be performed as a same-day procedure. In 2005, the United States Foods and Drugs Administration (FDA) approved the pivotal trial of the TEVAR for treating the patients with thoracic aorta aneurysm (2).

Certainly, blunt traumatic thoracic aortic injuries are among the most dangerous and fatal emergency situations. The typical mechanism causing these injuries is blunt deceleration, usually from motor vehicle collisions, falls, and crushes with significant amounts of force. So, the blunt traumatic thoracic aortic injuries occur in young patients with multiple traumas (3). Since the last decade, so important advancements have been developed in medical and surgi- cal management of blunt traumatic thoracic aortic injuries. Endovascular stent graft technologies are employed increasingly as an off label emergency treatment of these kinds of aortic injuries $(4,5)$. Finally, as experiments, we present two cases of 23 and 25 year-old men, who were admitted to our hospital because of blunt traumatic thoracic aortic injuries from car accidents. They were operated by the TEVAR technique. The management of both patients was the same. First of all, a chest X-ray was performed. The widening of mediastinum was obvious on the X-ray (Figure 1).

In the next step, Computed Tomography (CT) angiography and an aortography were done.

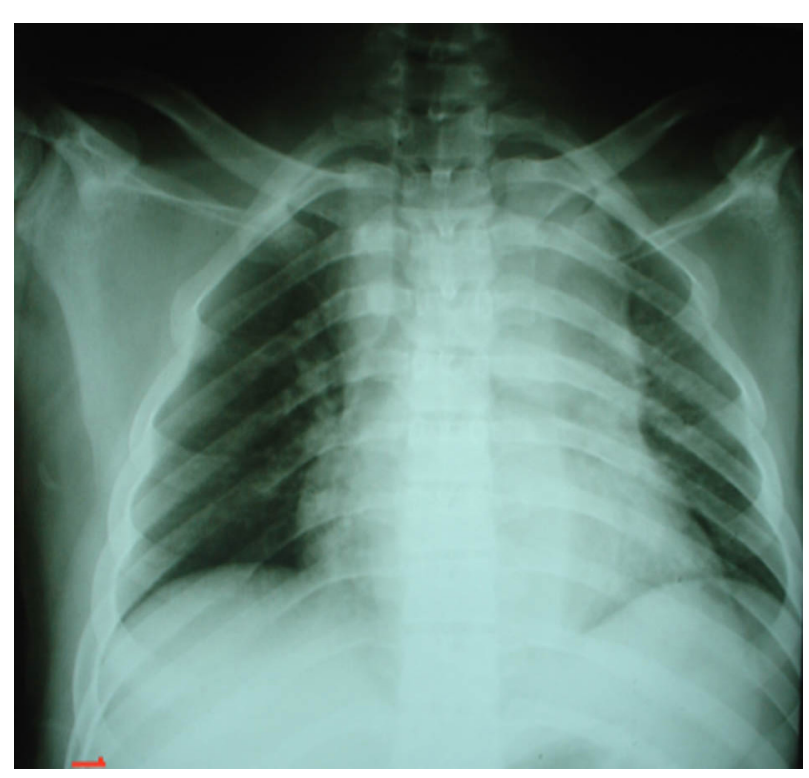

Figure 1. Preoperation CXR-widening of mediastinum is obvious in preoperation CXR. 
This study confirms the dissection of descending thoracic aorta. The patients were underwent the repair of thoracic aorta by the EV stent insertion approach. At the end of surgery, after repairing the injured part of aorta, the patients were referred to Intensive Care Unit (ICU) in the surgery ward. They were closely observed for 3 days. After three days of hospitalization, they were discharged with acceptable conditions.

As it is obvious, this minimally invasive EV approach results in several advantages for the patients compared with the open surgical repair. Therefore, it is highly recommended that the TEVAR be used instead of the open repair of blunt traumatic injury of the aorta.

\section{Footnotes}

Authors' Contribution:Mehrdad Vahedian developed the original idea and protocol and wrote the manuscript. Masumeh Taghizadeh collected the data and wrote the manuscript. Somayeh Rastinnia collected, abstracted, and analyzed data, wrote the manuscript, and was a guarantor.
Funding/Support:This study was supported by the surgery ward of Kerman University of Medical Sciences.

\section{References}

1. Cannon RM, Trivedi JR, Pagni S, Dwivedi A, Bland JN, Slaughter MS, et al. Open repair of blunt thoracic aortic injury remains relevant in the endovascular era. J Am Coll Surg. 2012;214(6):943-9. doi:10.1016/j.jamcollsurg.2012.03.003. [PubMed: 22541985]

2. Azizzadeh A, Keyhani K, Miller C3, Coogan SM, Safi HJ, Estrera AL. Blunt traumatic aortic injury: initial experience with endovascular repair. J Vasc Surg. 2009;49(6):1403-8. doi: 10.1016/j. jvs.2009.02.234. [PubMed:19497498]

3. Miller LE. Potential long-term complications of endovascular stent grafting for blunt thoracic aortic injury. ScientificWorldJournal. 2012;2012:897489. doi: 10.1100/2012/897489. [PubMed: 22547999]

4. Rousseau H, Elaassar O, Marcheix B, Cron C, Chabbert V, Combelles $\mathrm{S}$, et al. The role of stent-grafts in the management of aortic trauma. Cardiovasc Intervent Radiol. 2012;35(1):2-14. doi: 10.1007/s00270-011-0135-9. [PubMed: 21442377]

5. Borsa JJ, Hoffer EK, Karmy-Jones R, Fontaine AB, Bloch RD, Yoon $J K$, et al. Angiographic description of blunt traumatic injuries to the thoracic aorta with specific relevance to endograft repair. $J$ Endovasc Ther. 2002;9 Suppl 2:II84-91. [PubMed:12166847] 